\title{
Increased serum thromboxane A2 and prostacyclin but lower complement C3 and C4 levels in COVID-19: associations with chest CT-scan anomalies and lowered peripheral oxygen saturation
}

\author{
Hussein Kadhem Al-Hakeim ${ }^{\text {a }}$, Shaymaa Ali Al-Hamami, ${ }^{\text {, }}$, Michael Maes ${ }^{\text {c, d, e }}$ \\ a Department of Chemistry, College of Science, University of Kufa, Iraq. E-mail: \\ headm2010@yahoo.com \\ ${ }^{\mathrm{b}}$ Department of Medical Laboratory Techniques, Altoosi University College, Najaf, Iraq. E-mail: \\ shaima.alhmmamy@altoosi.edu.iq \\ ${ }^{\mathrm{c}}$ Department of Psychiatry, Medical University of Plovdiv, Plovdiv, Bulgaria. \\ d Department of Psychiatry, Faculty of Medicine, Chulalongkorn University, Bangkok, Thailand. \\ e School of Medicine, IMPACT Strategic Research Centre, Deakin University, PO Box 281, \\ Geelong, VIC, 3220, Australia. \\ Corresponding author: \\ Prof. Dr. Michael Maes, M.D., Ph.D., \\ IMPACT Strategic Research Center, \\ Barwon Health, \\ Deakin University, \\ Geelong, Vic, Australia. \\ E-mail: dr.michaelmaes@hotmail.com. \\ https//:scholar.google.co.th/citations?user=1wzMZ7UAAAAJ\&hl=th\&oi=ao
}

\begin{abstract}
Background. COVID-19 patients suffer from hypercoagulation and activated immuneinflammatory pathways. This study was performed to assay serum complement $\mathrm{C} 3$ and $\mathrm{C} 4$, and thromboxane A2 (TxA2) and prostacyclin (PGI2) in association with chest CT scan anomalies (CCTAs) and peripheral oxygen saturation (SpO2)

Methods. Serum levels of C3, C4, TxA2, and PGI2 were measured by ELISA and albumin, calcium, and magnesium by spectrophotometric method in 60 COVID-19 patients and 30 controls.

Results. C3 and C4 are significantly decreased and TxA2 and PGI2 significantly increased in COVID-19 patients as compared with controls. Neural networks showed that a combination of $\mathrm{C} 3$, albumin, and TxA 2 yielded a predictive accuracy of $100 \%$ in detecting COVID-19 patients. SpO2 was significantly decreased in COVID-19 patients and was inversely associated with TxA2 and PGI2, and positively with C3, C4, albumin, and calcium. CCTAs were accompanied by lower $\mathrm{SpO} 2$ and albumin, and increased PGI2 levels. Patients with positive IgG results show significantly higher SpO2, TxA2, PGI2, and $\mathrm{C} 4$ levels than IgG negative patients.

Conclusion. Hypoalbuminemia, which is strongly associated with lung lesions and lowered peripheral oxygen saturation, is characterized by increased TxA2, suggesting that interactions between immune-inflammatory pathways and platelet hyperactivity participate in the pathophysiology of COVID-19 and consequently may play a role in
\end{abstract}


enhanced risk of hypercoagulability and venous thromboembolism. These mechanisms are aggravated by lowered calcium and magnesium levels.

Keywords: COVID-19, C3, C4, inflammation, cytokines, biomarkers, thromboxane A2, prostacyclin.

\section{Introduction}

The clinical spectrum of SARS-CoV-2 infection ranges from asymptomatic infection to mild upper respiratory tract disease to severe viral pneumonia with respiratory failure and even death [1]. COVID-19 patients frequently suffer from major symptoms, including acute respiratory distress syndrome (ARDS), as well as inflammation with a possible cytokine storm, hypercoagulation, and thrombosis [2-4].

The COVID-19 virus enters the lung cells after binding of viral Spike proteins-S with the ACE2 receptors [5] and, consequently, the virus may cause histopathological lesions, which appear to be similar to those observed in other forms of ARDS [6]. Coronaviruses activate complement pathways which are a major component of innate immunity and act to remove invading pathogens [7]. Complement activation may result in immune-mediated lung damage [8] and is central to the pathophysiology of ARDS [9] and severe COVID-19 disease, which often resembles complementopathies [7]. Activation of the complement system leads to proteolytic cleavage of the key complement molecules $\mathrm{C} 3$ and $\mathrm{C} 4$ [10] leading to cleavage products including $\mathrm{C} 3 \mathrm{a}, \mathrm{C} 3 \mathrm{~b}, \mathrm{C} 4 \mathrm{a}$, and $\mathrm{C} 4 \mathrm{~b}$, which may trigger inflammatory cell recruitment and neutrophil activation [11]. Ghazavi et al. (2020) detected increased C3 and C4 complement levels in non-severe COVID-19, but lowered levels in severe COVID-19 patients which could be explained by increased consumption through formation of immune complexes [12]. Low serum C3 levels are detected in critically ill COVID-19 patients and are associated with a poor prognosis [13].

The severity of COVID-19 symptoms including end-organ damage is caused by an overzealous inflammatory response in part associated with complement activation, endothelial injury, neutrophil activation, thrombophilia, hypercoagulability, and thrombotic microangiopathy [7, 14-16]. About one third of ICU patients with COVID-19 have thrombotic complications, of which venous thromboembolic events are the most common [17]. The association between complement activation and coagulation mechanisms may cause life-threatening complications and as such the complementcoagulation network is an important drug target [18]. Nevertheless, only few studies in COVID-19 focused on C3 and C4 levels in relation to thromboxane A2 (TxA2) and prostacyclin (PGI2).

Endogenous $\mathrm{TxA}_{2}$, which is synthesized from arachidonic acid via cyclooxygenase (COX)-1, COX-2, and TxA2 synthase (TxAS), is produced by activated platelets and exerts prothrombotic effects [19]. TxA2 binds to the prostanoid thromboxane receptor, which triggers the binding to G-proteins thereby mediating calcium signaling and facilitating platelet aggregation and vasoconstriction [20,21]. COX-1, constitutively expressed in platelets, is a dominant source of TxA2 biosynthesis in humans [22]. In COVID-19, interleukin-1 (IL-1), a pro-inflammatory cytokine, stimulates TxA2 production [23]. PGI2 is mainly produced by endothelial and vascular smooth muscle cells [24] via COX-2 [25]. While TxA2 production causes platelet aggregation and vasoconstriction, PGI2 inhibits 
platelet aggregation and induces vascular smooth muscle relaxation and endotheliumrelated vasodilation [26-28]. The endothelial dysfunction following SARS-CoV-2 infection may be caused by lowered endothelial nitric oxide synthase activity and nitric oxide production and VEGF release following systemic hypoxia, whilst PGI2 may enhance angiogenesis and tissue repair through increased VEGF [29, 30].

Recently, we published that chest CT abnormalities (CCTAs) (comprising groundglass opacities (GGOs), pulmonary densification areas consistent with residual lesions, pneumonic consolidation, and crazy-paving patterns), could be observed in $78.3 \%$ of RTPCR test-proven COVID-19 cases and that the presence of CCTAs was characterized by significantly lowered peripheral oxygen saturation ( $\mathrm{SpO} 2)$ and serum levels of albumin [31]. The latter is a negative acute phase protein which is lowered in response to the systemic inflammatory response in COVID-19 [1, 31-33]. Moreover, lowered SpO2 values were significantly associated with signs of immune activation, and positively with albumin, magnesium, and calcium [31]. In addition, the latter study found that lowered serum calcium was the single best biomarker of acute COVID-19 and was more important than inflammatory biomarkers including interleukin-6 (IL-6) and C-reactive protein (CRP) in discriminating COVID-19 patients from healthy controls. We have argued that beta coronavirus-mediated calcium dyshomeostasis is due to a) hypoalbuminemia with around $45 \%$ of calcium being bound to albumin [34]; and b) to activation of store-operated calcium entry (SOCE) channels by endoplasmic-reticulum stress $[35,36]$, which is a consequence of infections with those viruses [37, 38]. Magnesium has antioxidant [39], antiinflammatory [40] and antithrombotic [41] properties with about one third of total magnesium levels being bound to albumin [42].

The present study was conducted to examine the associations between immuneinflammatory (as measured with albumin, C3 and C4) and thrombosis-related (TxA2 and $\mathrm{PGI} 2)$ biomarkers in relation to $\mathrm{SpO} 2$ and CCTAs

\section{Subjects and Methods}

\section{Subjects}

The present study recruited sixty patients with confirmed acute SARS-CoV-2 infection and 30 normal controls. The patients were recruited at the Al-Amal Specialized Hospital for Communicable Diseases and Al-Sadr Teaching Hospital in Najaf governorateIraq between September and November 2020. The diagnosis of SARS-CoV-2 infection was based on positive test results of COVID-19 nucleic acids by reverse transcription realtime polymerase chain reaction (rRT-PCR) and positive IgM to SARS-CoV-2, and symptoms of acute infectious disease including fever, fatigue, dyspnea, dry cough, dysgeusia, and anosmia. Patients were excluded for the presence of premorbid medical disease including diabetes, liver disease, chronic kidney disease, neurodegenerative and neurologic disorders including multiple sclerosis, and Parkinson's and Alzheimer's disease.

Chest computed tomography (CT) scans were used to examine chest CT abnormalities (CCTAs), comprising GGOs, pulmonary densification areas consistent with residual lesions, pneumonic consolidation, and crazy-paving patterns [43]. We divided the patient group into those with (COVID+CCTA) and without (COVID-CCTA) CCTAs. The patients were further divided according to $\mathrm{IgG}$ results into negative- $\operatorname{IgG}$ and positive- $\operatorname{IgG}$ 
subgroups to examine the difference in the measured biomarkers between these subgroups. We also recruited 30 healthy controls, age, and sex-matched to the patient groups. All controls were free from any systemic disease. However, as a public method to enhance their immunity against COVID-19 infection, some healthy controls were taken zinc and vitamins $\mathrm{C}$ and $\mathrm{D}$.

The IRB of the University of Kufa has approved the study (617/2020). All controls and patients gave written informed consent before participation in this study. The study was conducted according to Iraq and international ethics and privacy laws and was conducted ethically in accordance with the World Medical Association Declaration of Helsinki. Furthermore, our IRB follows the International Guideline for Human Research protection as required by the Declaration of Helsinki, The Belmont Report, CIOMS Guideline and International Conference on Harmonization in Good Clinical Practice (ICHGCP).

\section{Measurements}

Upon admission of the patient into hospital, RT-PCR tests were conducted using the Lyra ${ }^{\circledR}$ Direct SARS-CoV-2 Assay kits (Quidel Corporation, CA, USA) using the Applied Biosystems ${ }^{\circledR}$ QuantStudio ${ }^{\text {TM }} 5$ Real-Time PCR System (Thermo Fisher Scientific; Life Technologies Holdings Pte Ltd., Marsiling Industrial Estate, Singapore). The Lyra ${ }^{\circledR}$ Direct SARS-CoV-2 assay kit is a real-time RT-PCR assay for the qualitative detection of human coronavirus SARS-CoV-2 from viral RNA extracted from nasal, nasopharyngeal or oropharyngeal swab specimens.

Upon inclusion in the study, fasting blood samples were taken in the early morning directly after awakening. Five milliliters of venous blood samples were drawn and transferred into clean plain tubes. After ten minutes, the clotted blood samples were centrifuged for five minutes at $3000 \mathrm{rpm}$, and then serum was separated and transported into three new Eppendorf tubes until assay. Hemolyzed samples were rejected. Serum C3, C4, PGI2, and TxA2 were measured using ELISA techniques based on sandwich technique using ready-for-use kits supplied by Melsin Medical Co (Jilin, China). The inter-assay CV values of all assays were less than $12 \%$. Serum albumin, magnesium, and total calcium were measured spectrophotometrically by kits supplied by Biolabo ${ }^{\circledR}$ (Maizy, France). The procedures were followed exactly without modifications according to the manufacturer's instructions. A qualitative ACON ${ }^{\circledR}$ COVID-19 IgG/IgM rapid test was used to detect IgG and IgM in all subjects' sera. The kits have a sensitivity $\geq 99.1 \%$ and a specificity $\geq$ of $98.2 \%$.

\section{Statistical analysis}

The group-differences among continuous variables were examined by analysis of variance (ANOVA), while associations between nominal variables were checked using analysis of contingency tables $\left(\chi^{2}\right.$-test). Pearson's product-moment and Spearman's rankorder correlation coefficients were used to determine the correlations between biomarkers and clinical and cognitive scores. To assess the associations between diagnosis and biomarkers, we used multivariate general linear models (GLM) while adjusting for confounding variables such as tobacco use disorder (TUD), age, sex, and BMI. Consequently, we used tests for between-subject effects to determine the relationships between diagnosis and the separate biomarkers. The effect size was estimated using partial 
eta-squared values. We also computed estimated marginal mean (SE) values provided by the GLM analysis and performed protected pairwise comparisons among treatment means. Binary logistic regression analysis was employed to determine the best predictors of COVID-19 versus the control group. Odd's ratios with $95 \%$ confidence intervals were computed as well as Nagelkerke values which were used as pseudo- $\mathrm{R}^{2}$ values. We used multiple-regression analysis to delineate the significant biomarkers predicting symptom domains while allowing for the effects of age, gender, and education. All regression analyses were tested for collinearity using tolerance and VIF values. All tests were twotailed, with a p-value of 0.05 used to determine statistical significance.

Neural network analysis was conducted with diagnosis (COVID-19 versus controls) as output variables and biomarkers as input variables as explained previously [44]. In brief: an automated feed-forward architecture, multilayer perceptron neural network model was employed to check the associations between biomarkers (input variables) and the diagnosis of COVID-19 versus controls (output variables). We trained the model with two hidden layers with up to 4 nodes in each layer, 20-50 epochs, minibatch training with gradient descent. One consecutive step with no further decrease in the error term was used as stopping rule. We extracted three samples: a) a holdout sample $(33.3 \%)$ to check the accuracy of the final network; b) a training sample $(47.7 \%)$ to estimate the network parameters, and c) a testing sample (20.0\%) to prevent overtraining. We computed error, relative error, and importance and relative importance of all input variables were computed. IBM SPSS windows version 25, 2017 was used for all statistical analysis.

\section{Results}

\section{Socio-demographic data}

Table 1 shows the socio-demographic and clinical data in COVID-19 patients and the healthy control (HC) group. There was no significant difference between study groups in age, BMI, education, residency, marital status, and TUD. Sixty patients were recruited to participate, namely admission room: 35 patients, ICU: 16 patients, and RCU: 9 patients. All patients were on $\mathrm{O}_{2}$ therapy, and were administered paracetamol, bromhexine, vitamin C, vitamin D, and zinc. Thirty-six patients out of 60 were IgG positive.

\section{Differences in biomarkers among groups}

The results of the biomarkers in COVID-19 patients compared with the control group are presented in Table 2. There was a significant decrease in $\mathrm{SpO}_{2}$ in COVID+CCLA patients compared to COVID-CCLA patients and controls. There was also a significant decrease in serum albumin levels in COVID-19 patients compared to controls, with the lowest levels in the COVID+CCLA group. Serum magnesium was not significantly different between the three study groups. Serum PGI2 and TxA2 showed a significant increase in COVID-19 patients compared with controls, with the highest levels in the COVID+CCLA group. The significant increases in TxA2 in COVID-19 remained significant after forced entry of albumin $(\mathrm{F}=6.93, \mathrm{df}=2 / 86, \mathrm{p}=0.002)$, and albumin and prostacyclin $(\mathrm{F}=3.33$, df-2/85, $\mathrm{p}=0.040)$. Serum $\mathrm{C} 3, \mathrm{C} 4$, and total calcium were significantly lower in both patient groups when compared with controls. The differences in serum total calcium were no longer significant after covarying for albumin levels $(\mathrm{F}=0.16, \mathrm{df}=1 / 86, \mathrm{p}=0.850)$. 
Table 1. Socio-demographic and clinical data of COVID-19 patients and healthy controls (HC).

\begin{tabular}{|l|c|c|c|c|c|}
\hline Variables & $\begin{array}{c}\mathrm{HC} \\
(\mathrm{n}=30)\end{array}$ & $\begin{array}{c}\text { COVID-19 } \\
(\mathrm{n}=60)\end{array}$ & F/FEPT/ $\chi^{2}$ & $\mathrm{df}$ & $\mathrm{p}$ \\
\hline Age (years) & $40.1 \pm 8.8$ & $41.0 \pm 10.2$ & 0.17 & $1 / 88$ & 0.681 \\
\hline BMI (kg/m $\left.{ }^{2}\right)$ & $26.05 \pm 4.02$ & $27.07 \pm 3.62$ & 1.50 & $1 / 88$ & 0.225 \\
\hline Sex (Female/Male) & $6 / 24$ & $17 / 43$ & 0.73 & 1 & 0.393 \\
\hline Urban/Rural & $28 / 2$ & $52 / 8$ & - & - & 0.486 \\
\hline Single/married & $10 / 20$ & $17 / 43$ & 0.24 & 1 & 0.626 \\
\hline TUD (No/Yes) & $20 / 10$ & $39 / 21$ & 0.03 & 1 & 0.875 \\
\hline Employment (No/Yes) & $9 / 21$ & $21 / 39$ & 0.23 & 1 & 0.635 \\
\hline Education (years) & $10.7 \pm 3.2$ & $9.8 \pm 4.0$ & 1.07 & $1 / 88$ & 0.303 \\
\hline Admission room/ICU/RCU & - & $35 / 16 / 9$ & - & - & - \\
\hline Zinc (No/Yes) & $23 / 7$ & $1 / 59$ & 57.53 & 1 & $<0.001$ \\
\hline Vitamin D (No/Yes) & $27 / 3$ & $0 / 60$ & 77.14 & 1 & $<0.001$ \\
\hline Vitamin C (No/Yes) & $23 / 7$ & $0 / 60$ & 61.79 & 1 & $<0.001$ \\
\hline Dexamethasone (No/Yes) & & $9 / 51$ & & & \\
\hline Azithromycin (No/Yes) & & $25 / 35$ & & & \\
\hline Enoxaparin (No/Yes) & $28 / 32$ & & & \\
\hline O2 therapy (No/Yes) & & $0 / 60$ & & & $<0.001$ \\
\hline Bromhexine (No/Yes) & & $21 / 39$ & & & \\
\hline Paracetamol (No/Yes) & & $23 / 60$ & & & \\
\hline Omeprazole (No/Yes) & & $35 / 25$ & & & \\
\hline Ceftriaxone (No/Yes) & & $36 / 24$ & & & \\
\hline IgG (Positive/Negative) & & & & & \\
\hline
\end{tabular}

Results are shown as mean \pm SD, FEPT: Fisher's exact probability test, BMI: body mass index, TUD: tobacco use disorder

Table 2. Biomarkers in COVID-19 patients divided into those with chest CT scan abnormalities (COVID+CCTA) and those without CCTA (COVID-CCTA) and healthy controls (HC).

\begin{tabular}{|c|c|c|c|c|c|c|}
\hline Biomarkers & $\begin{array}{c}\mathrm{HC}^{\mathrm{A}} \\
(\mathrm{n}=\mathbf{3 0})\end{array}$ & $\begin{array}{c}\text { COVID-CCTA } \\
(n=15)\end{array}$ & $\begin{array}{c}\text { COVID+CCTA } \\
(n=45)\end{array}$ & $\mathbf{F}$ & df & $\mathbf{p}$ \\
\hline $\mathrm{SPO}_{2}$ & $98.53 \pm 0.68^{\mathrm{C}}$ & $97.13 \pm 0.74^{\mathrm{C}}$ & $92.62 \pm 3.40^{\mathrm{A}, \mathrm{B}}$ & 56.37 & $2 / 87$ & $<0.001$ \\
\hline Albumin $\quad \mathrm{g} / 1$ & $43.10 \pm 3.11^{\mathrm{B}, \mathrm{C}}$ & $33.47 \pm 5.08^{\text {A, C }}$ & $29.71 \pm 3.79^{\mathrm{A}, \mathrm{B}}$ & 111.12 & $2 / 87$ & $<0.001$ \\
\hline Magnesium $\mathrm{mM}$ & $0.933 \pm 0.102^{\mathrm{C}}$ & $1.018 \pm 0.223$ & $1.010 \pm 0.172^{\mathrm{A}}$ & 2.42 & $2 / 87$ & 0.005 \\
\hline Calcium $\mathrm{mM}$ & $2.264 \pm 0.096^{\mathrm{B}, \mathrm{C}}$ & $2.089 \pm 0.148^{\mathrm{A}}$ & $2.021 \pm 0.128^{\mathrm{A}}$ & 36.02 & $2 / 87$ & $<0.001$ \\
\hline Thromboxane A2 pg/ml & $236.4 \pm 64.6^{\mathrm{B}, \mathrm{C}}$ & $337.9 \pm 64.1^{\mathrm{A}}$ & $366.0 \pm 98.7^{\mathrm{A}}$ & 22.17 & $2 / 87$ & $<0.001$ \\
\hline Prostacyclin $\mathrm{pg} / \mathrm{ml}$ & $104.7 \pm 36.9^{\mathrm{B}, \mathrm{C}}$ & $153.6 \pm 45.4^{\mathrm{A}, \mathrm{C}}$ & $188.7 \pm 45.0^{\mathrm{A}, \mathrm{B}}$ & 35.00 & $2 / 87$ & $<0.001$ \\
\hline Complement C3 mg/l & $872.1 \pm 285.5^{\mathrm{B}, \mathrm{C}}$ & $346.7 \pm 134.0^{\mathrm{A}}$ & $302.9 \pm 88.2^{\mathrm{A}}$ & 92.00 & $2 / 87$ & $<0.001$ \\
\hline Complement $\mathrm{C} 4 \mathrm{mg} / \mathrm{l}$ & $381.7 \pm 138.7^{\mathrm{B}, \mathrm{C}}$ & $266.3 \pm 110.0^{\mathrm{A}}$ & $273.1 \pm 87.3^{\mathrm{A}}$ & 9.98 & $2 / 87$ & $<0.001$ \\
\hline
\end{tabular}

All results are shown as mean (SE). All results of univariate GLM analyses examining the associations between diagnosis (healthy control and COVID-19 patients divided into two groups depending on the presence or absence of CCTA) after adjusting for age, sex, tobacco use disorder, and body mass index.

$\mathrm{SPO}_{2} \%$ : oxygen saturation percentage. 
Multivariate differences between COVID-19 patients and controls.

Using lowered levels of $\mathrm{SpO} 2, \mathrm{C} 3$ and albumin in a binary logistic regression analysis, we found a significant discrimination between COVID-19 patients and controls with an accuracy of $100 \%$. Nevertheless, the SPSS program does not allow to estimate the regression parameters in this condition. Table 3 shows the network information of a neural network model which examines the discrimination of COVID-19 versus controls. The network has been trained using one hidden layer with 4 units in layer 1. Hyperbolic tangent was used as an activation function in hidden layer 1 and identity in the output layer. The partitioned confusion matrix showed an AUC ROC $=1.000$ with an accuracy of $100.0 \%$ in the holdout sample and a sensitivity of $100.0 \%$ and specificity of $100.0 \%$. Figure 1 shows the relative importance of the most effective input variables (C3, albumin, TxA2, C4, PGI2, and $\mathrm{SPO} 2$ ) that represent the most important determinants of the model's predictive power.

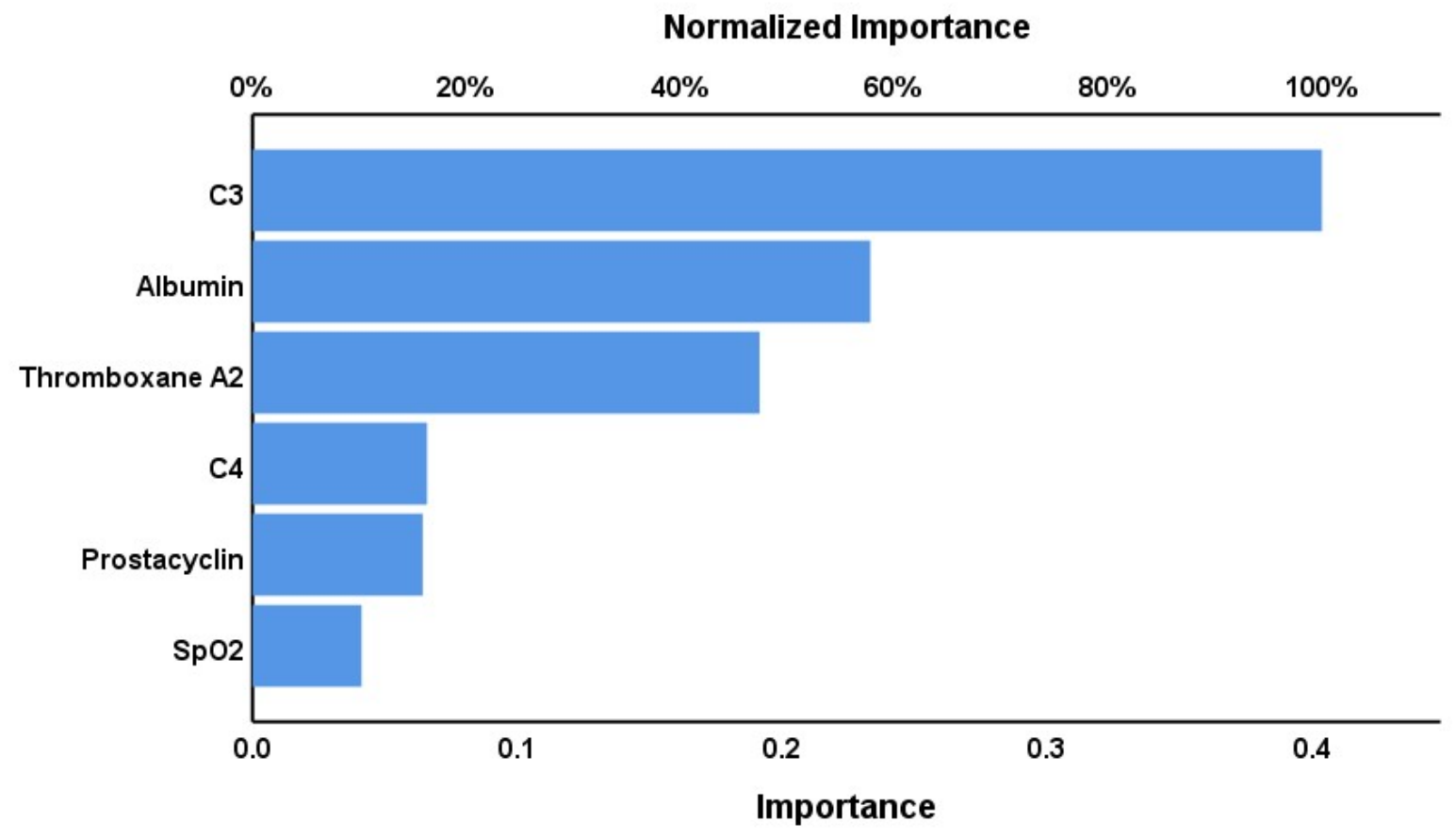

Figure 1. Results of neural network (importance chart) with diagnosis of COVID-19 versus controls as output variables and biomarkers as input variables.

Table 3. Results of neural networks with diagnosis of COVID-19 versus heathy controls (HC) as output variables and biomarkers as input variables.

\begin{tabular}{|l|l|c|}
\hline & Model & COVID-19 vs. HC \\
\hline \multirow{3}{*}{ Input Layer } & Number of units & 6 \\
\cline { 2 - 3 } & Rescaling method & Normalized \\
\hline \multirow{3}{*}{ Hidden layers } & Number of hidden layers & 1 \\
\cline { 2 - 3 } & Number of units in hidden layer 1 & 4 \\
\cline { 2 - 3 } & Activation Function & Hyperbolic tangent \\
\hline \multirow{3}{*}{ Output layer } & Dependent variables & COVID-19 vs. HC \\
\cline { 2 - 3 } & Number of units & Identity \\
\cline { 2 - 3 } & Activation function & \\
\hline
\end{tabular}




\begin{tabular}{|l|l|c|}
\hline \multirow{4}{*}{ Training } & Error function & Sum of squares \\
\hline \multirow{5}{*}{ Testing } & Sum of squares error term & 0.420 \\
\cline { 2 - 3 } & \% incorrect or relative error & $0.0 \%$ \\
\cline { 2 - 3 } & Prediction (sens, spec) & $100 \%, 100 \%$ \\
\hline \multirow{2}{*}{ Holdout } & Sum of Squares error & 0.252 \\
\cline { 2 - 3 } & \%incorrect or relative error & $0.0 \%$ \\
\cline { 2 - 3 } & Prediction (sens spec) & $100 \%, 100 \%$ \\
\cline { 2 - 3 } & AUC ROC & 1.00 \\
\cline { 2 - 3 } & \%incorrect or relative error & $0.0 \%$ \\
\cline { 2 - 3 } & Prediction (sens, spec) & $100 \%, 100 \%$ \\
\hline
\end{tabular}

AUC ROC: area under curve of receiver operating curve, sens: sensitivity, spec: specificity.

Associations of biomarkers with anti-SARS-CoV-2 IgG antibodies, CCTAs and SpO2.

Table 4 shows the biomarkers in COVID-19 patients with positive versus negative anti-SARS-CoV-2 IgG antibody titers. The positive IgG group showed a significant increase in $\mathrm{SpO} 2$, TxA2, PGI2, and $\mathrm{C} 4$ compared with the patients with negative IgG antibodies. These differences remained significant after FDR p-correction (at $p=0.016$ ). The same table shows that $\mathrm{SpO} 2$ and albumin were significantly lower and prostacyclin significantly higher in COVID+CCTA as compared with COVID-CCTA patients. These differences remained significant after FDR p-correction (at $\mathrm{p}=0.033$ ).

Table 5 shows the intercorrelation matrix between $\mathrm{SpO} 2$ and the biomarkers. $\mathrm{SpO} 2$ was significantly and negatively correlated with TxA2 and prostacyclin, and positively with C3, C4, albumin, and calcium. TxA2 was significantly and positively correlated with prostacyclin and negatively with $\mathrm{C} 3$, albumin, and calcium. Prostacyclin levels were significantly and positively correlated with magnesium and inversely with $\mathrm{C} 3$, albumin, and calcium.

Table 6 shows the regression of TxA2 on immune-inflammatory biomarkers. The first regression shows that $39.3 \%$ of the variance in TxA2 was explained by the regression on albumin (inversely) and prostacyclin (positively). The second regression shows that $42.0 \%$ of the variance in TxA2 was explained by the regression on C3 (inversely) and C4 and prostacyclin (both positively). 
Table 4. Differences in biomarkers between COVID-19 patients with and without antiSARS-CoV-2 IgG antibodies and with and without chest CT scan anomalies (CCTAs).

\begin{tabular}{|c|c|c|c|c|c|}
\hline Biomarker & $\begin{array}{c}\begin{array}{c}\text { Negative IgG } \\
\mathbf{N}=\mathbf{2 4}\end{array} \\
\end{array}$ & $\begin{array}{c}\begin{array}{c}\text { Positive IgG } \\
\mathrm{N}=\mathbf{3 6}\end{array} \\
\end{array}$ & $\mathbf{F}$ & df & $\mathbf{P}$ \\
\hline $\mathrm{SpO}_{2}$ & $92.25 \pm 4.31$ & $94.75 \pm 2.56$ & 7.97 & $1 / 58$ & 0.007 \\
\hline Albumin & $29.83 \pm 4.37$ & $31.19 \pm 4.43$ & 1.37 & $1 / 58$ & 0.246 \\
\hline Magnesium $\mathrm{mM}$ & $1.017 \pm 0.201$ & $1.009 \pm 0.174$ & 0.02 & $1 / 58$ & 0.880 \\
\hline Calcium $\mathrm{mM}$ & $2.012 \pm 0.132$ & $2.056 \pm 0.137$ & 1.54 & $1 / 58$ & 0.219 \\
\hline Thromboxane A2 pg/ml & $306.3 \pm 57.6$ & $394.1 \pm 93.8$ & 16.78 & $1 / 58$ & $<0.001$ \\
\hline Prostacyclin $\mathrm{pg} / \mathrm{ml}$ & $160.2 \pm 40.9$ & $193.1 \pm 47.2$ & 7.78 & $1 / 58$ & 0.007 \\
\hline Complement C3 mg/l & $329.0 \pm 120.2$ & $303.7 \pm 88.4$ & 0.88 & $1 / 58$ & 0.351 \\
\hline Complement C4 mg/l & $227.8 \pm 81.1$ & $300.4 \pm 89.2$ & 10.23 & $1 / 58$ & 0.002 \\
\hline Biomarkers & $\begin{array}{c}\text { No CCTAs } \\
n=15\end{array}$ & $\begin{array}{c}\text { CCTAs } \\
n=45\end{array}$ & $\mathbf{F}$ & df & $\mathbf{p}$ \\
\hline $\mathrm{SpO}_{2}$ & $97.13 \pm 0.74$ & $92.62 \pm 3.40$ & 25.94 & $1 / 58$ & $<0.001$ \\
\hline Albumin & $33.47 \pm 5.08$ & $29.71 \pm 3.79$ & 9.25 & $1 / 58$ & 0.004 \\
\hline Magnesium $\mathrm{mM}$ & $1.0178 \pm 0.233$ & $1.010 \pm 0.172$ & 0.02 & $1 / 58$ & 0.895 \\
\hline Calcium $\quad \mathrm{mM}$ & $2.089 \pm 0.147$ & $2.038 \pm 0.135$ & 2.94 & $1 / 58$ & 0.092 \\
\hline Thromboxane A2 pg/ml * & $337.9 \pm 64.1$ & $366.0 \pm 98.7$ & 1.09 & $1 / 58$ & 0.301 \\
\hline Prostacyclin $\mathrm{pg} / \mathrm{ml}$ & $153.6 \pm 45.4$ & $188.7 \pm 45.0$ & 6.81 & $1 / 58$ & 0.011 \\
\hline Complement C3 mg/1 ** & $346.7 \pm 134.0$ & $302.9 \pm 88.2$ & 1.92 & $1 / 58$ & 0.171 \\
\hline Complement C4 mg/l & $266.3 \pm 110.0$ & $273.4 \pm 87.3$ & 0.59 & $1 / 58$ & 0.809 \\
\hline
\end{tabular}

All results of univariate GLM analysis; results are shown as mean \pm SE.

*Processed in Ln and ** square root transformation

$\mathrm{SpO}_{2} \%$ : oxygen saturation percentage.

Table 5. Intercorrelation matrix between oxygen saturation percentage $(\mathrm{SpO} 2)$ and different biomarkers of COVID-19.

\begin{tabular}{|l|c|c|c|}
\hline Biomarkers & SpO2 & Thromboxane A2 & Prostacyclin \\
\hline Thromboxane A2 & $-0.362(0.001)$ & - & - \\
\hline Prostacyclin & $-0.380(0.001)$ & $0.539(0.002)$ & - \\
\hline Complement C3 & $0.598(0.001)$ & $-0.544(0.002)$ & $-0.593(0.001)$ \\
\hline Complement C4 & $0.355(0.001)$ & $0.028(0.800)$ & $-0.014(0.9)$ \\
\hline Albumin & $0.617(0.001)$ & $-0.549(0.002)$ & $-0.572(0.001)$ \\
\hline Magnesium & $-0.208(0.055)$ & $0.178(0.115)$ & $0.308(0.005)$ \\
\hline Calcium & $0.490(0.001)$ & $-0.379(0.002)$ & $-0.496(0.001)$ \\
\hline
\end{tabular}

All results of partial correlation analysis after covarying for age, sex, body mass index and tobacco use disorder. Shown are false discovery rate corrected $\mathrm{p}$ values. 
Table 6. Results of multiple regression analysis with PxA2 as dependent variable and immune-inflammatory mediators and prostacyclin as explanatory variables.

\begin{tabular}{|c|c|c|c|c|c|c|c|c|}
\hline Dependent Variables & Explanatory Variables & $\boldsymbol{\beta}$ & $\mathbf{t}$ & $\mathbf{p}$ & F model & df & $\mathbf{p}$ & $\mathbf{R}^{2}$ \\
\hline \multirow{3}{*}{$\# 1 . \mathbf{T x A 2}$} & Model & & & & 28.13 & $2 / 87$ & $<0.001$ & 0.393 \\
\hline & Albumin & -0.387 & -3.923 & $<0.001$ & & & & \\
\hline & Prostacyclin & 0.328 & 3.319 & 0.001 & & & & \\
\hline \multirow{4}{*}{$\# 2$. TxA2 } & Model & & & & 20.78 & $3 / 86$ & $<0.001$ & 0.420 \\
\hline & $\mathrm{C} 3$ & -0.525 & -4.471 & $<0.001$ & & & & \\
\hline & $\mathrm{C} 4$ & 0.241 & 2.498 & 0.014 & & & & \\
\hline & Prostacyclin & 0.227 & 2.118 & 0.037 & & & & \\
\hline
\end{tabular}

\section{Discussion}

Changes in complement in COVID-19

The first major finding of the present study is that $\mathrm{C} 3$ and $\mathrm{C} 4$ are significantly decreased in COVID-19 patients. As reviewed in the introduction, there were some reports that C3 is significantly lowered in severe COVID-19 as compared with controls. This may be explained by increased cleavage during activation and increased consumption following immune complex formation [12]. C3 levels tend to increase gradually in recovered COVID-19 patients, whilst C3 levels were decreased in non-survivors and associated with increased risk of in-hospital death [13]. The levels of complement C4 were decreased from day 0 to day 10 in patients hospitalized for more than two weeks, but not in patients who were discharged earlier [45]. C3 polymorphism may be a significant risk factor of mortality due to COVID-19 [46]. However, in a previous analysis, no major variations in complement $\mathrm{C} 3$ or C4 levels were observed between severe and less severe COVID-19 study groups [47], whereas another report found increased C3 and C4 in COVID-19 patients [48].

We also found that lowered $\mathrm{SpO} 2$ is associated with lowered $\mathrm{C} 3$ and $\mathrm{C} 4$ levels. In this respect, it is interesting to note that, in COVID-19 patients, systemic complement activation is associated with respiratory failure [49]. Complement activation mediates in part the systemic immune-inflammatory response in SARS-CoV infection [8] and activation of complement C3 can worsen SARS-COV-related ARDS [50].

\section{Increased TxA2 and PGI2 in COVID-19}

The second major finding of this study is that TxA2 is significantly increased in COVID-19 patients when compared with controls. Platelets produce significant amounts of TxA2 and prostaglandins dependent upon the activity of COX-1, COX-2, and TxA2. On platelets, TxA2 binds to the prostanoid thromboxane receptor thereby initiating an amplification loop leading to further platelet activation, aggregation and TxA2 formation [51], which may, consequently, lead to a prothrombotic state with increased mortality risk $[17,52,53]$. Increased platelet activity and aggregability has been reported in patients with COVID-19 [54] and is associated with an increased mortality risk [55]. In addition, coagulopathies are often observed in COVID-19 with up to one-third of patients having thrombotic problems [56]. 
In our study, we observed a significant intertwined upregulation in TxA2 and PGI2 levels. Prostaglandins, including PGI2, are usually elevated in response to inflammatory or toxic stimuli [57]. Endothelial PGI2 binds to the Gs-coupled PGI2 receptor on platelets thereby reducing platelet reactivity, which can be critical to minimizing the risk for atherothrombotic events [58]. PGI2 signaling induces cytosolic cAMP thereby preventing platelet activation [59] and may reduce viral-induced illness by suppressing the induction of type-I interferons [60]. Moreover, PGI2 protects against cytokine toxicity by attenuating nuclear factor- $\kappa \mathrm{B}$ activity and possesses strong anti-inflammatory and immune-regulatory properties [61]. As such, increased levels of prostacyclin may attenuate the thrombotic and immune effects of increased TxA2. Nevertheless, in our study, we found that the increases in TxA2 in COVID-19 patients remained significant after adjusting for albumin and prostacyclin. In this regard it is important to note that PGI2 signaling may lead to an increased production of IL-6 from stromal cells [62] and may promote $\mathrm{T}$ helper-1 differentiation possibly through cAMP-PKA signaling [63].

The massive platelet activation in COVID-19 is probably not a direct consequence of the virus itself because SARS-CoV-2 has rarely been found in the serum of infected patients [64]. One of the mechanisms causing severe COVID-19 is believed to stem from an exaggerated immune-inflammatory response with complement-induced-coagulation, massive endothelial damage and systemic microangiopathy [65]. In severe COVID-19, widespread endothelial dysfunction and coagulopathies and complement-induced thrombosis may cause systemic microangiopathy and thromboembolism, which may lead to multi-organ failure thereby increasing the mortality rate [65]. Moreover, in COVID-19, activation of the alternative complement pathway is associated with microvascular injury and thrombosis [66]. Consequently, a pro-coagulative endothelium may induce endothelins thereby mediating infiltration of inflammatory cells in the lungs leading to ARDS in COVID-19 [67-69]. On the other hand, the endothelium mediates antithrombotic and antiinflammatory functions by releasing active endothelium-derived factors such as nitric oxide $\mathrm{PGI}_{2}[70]$.

\section{Lowered albumin, calcium, and magnesium in COVID-19}

In agreement with Al-Hakeim et al. (2020) and other studies reviewed in the latter paper [31], this study found that serum albumin, and total calcium and magnesium were significantly lowered in COVID-19. Hypoalbuminemia may be explained by the acute phase responses in COVID-19 with increased breakdown of albumin and increased production of positive acute phase proteins [71], and by enhanced capillary permeability leading to the leaking of albumin to the interstitial space [72]. Interestingly, in the current study we found a significant and inverse associations between TxA2, C3 and albumin levels, suggesting platelet hyperactivity - immune-inflammatory interactions in COVID19. A previous study showed that hypoalbuminemia, especially when serum albumin is $<$ $35 \mathrm{~g} / \mathrm{L}$, is associated with elevated D-dimers and enhanced risk of artery and venous thrombosis [73, 74]. The association between hypoalbuminemia and hypercoagulability and venous thromboembolism may be explained by the anticoagulant and antiplatelet activities of albumin [75]. Not only platelet-platelet but also platelet-leukocyte interactions play a key role in COVID-19 [76]. Activation of the prostanoid TxA2 receptor mediates not only thrombosis and angiogenesis, but also vascular inflammation [23]. In ARDS, 
platelets may function as effectors in immunity and inflammation [77, 78] and virusplatelet interactions increase thrombotic risk by fostering procoagulant and inflammatory states during viral infection [79]. The current study found that a combination of $\mathrm{C} 3$, albumin and TxA2 could be used as an extern validating criterion for the diagnosis of COVID-19. These data further underscore that the combined intertwined activities of immune-inflammatory and hemostasis pathways underpin the pathophysiology of COVID19.

Moreover, in agreement with Al-Hakeim et al. (2021), this study found that CCTAs are accompanied by lowered $\mathrm{SpO} 2$ and hypoalbuminemia, and additionally that lowered $\mathrm{SpO} 2$ is associated with low serum albumin [31]. Such findings indicate that the lesions caused by inflammatory lung damage may result in diminished lung oxygenation and systemic inflammation with hypoalbuminemia [31]. Interestingly, CCTAs are associated with the severity of COVID-19 [80] and are an important risk factor for myocarditis in COVID-19 patients [80].

As reviewed in our Introduction both calcium and magnesium are partially bound to albumin and therefore hypoalbuminemia may explain at least part of the lowered magnesium and calcium levels in COVID-19 [31]. Importantly, hypocalcemia is more common in severely ill COVID-19 patients than in mild cases, and may detect with high specificity the more critically ill patients or those with a poorer outcome $[81,82]$ and may predict hospitalization of COVID-19 patients [83].

\section{Biomarkers and $\operatorname{Ig} G$ positivity}

Another major finding is that patients with positive IgG titers showed higher SpO2, TxA2, PGI2 and C4 levels. Previously, we reported that patients with IgG positivity showed higher IL-6, sRAGE and ACE2 levels as compared to patients with negative IgG titers [31]. We found 60.0\% (the current study) and 66.7\% [31] of COVID-19 patients showed positive IgG levels, whereas a previous study reported that $77.9 \%$ of COVID-19 patients were IgG positive [84]. The antibody dynamics observed in COVID-19 patients are quite similar to the Ig responses in other viral infections, with an initial increase in IgM and increasing IgG levels when IgM starts to decrease [85]. Not only increased IL-6 may drive B-cell mediated IgG formation [86], but also complement activation promotes humoral immunity [87]. As we discussed previously, increased IgG titers are more pronounced in severe than in mild cases [88], and, therefore, the association between positive IgG titers and TxA2 and PGI2 found in the current study could reflect severity of illness.

\section{Conclusions}

COVID-19 is characterized by significantly decreased SpO2, C3 and C4 and significantly increased TxA2 and PGI2. A combination of C3, albumin, and TxA2 yielded a predictive accuracy of $100 \%$ in discriminating COVID-19 patients from healthy controls. $\mathrm{SpO} 2$ was significantly and positively associated with $\mathrm{C} 3, \mathrm{C} 4$, albumin, and calcium, and negatively with TxA2 and PGI2. Furthermore, CCTAs were accompanied by lower SpO2 and albumin, but higher prostacyclin. The strong association between CCPA-associated hypoalbuminemia and increased TxA2 suggests that intertwined interactions between 
immune-inflammatory pathways and platelet hyperactivity participate in the pathophysiology of COVID-19. These mechanisms may be aggravated by lowered calcium and magnesium, which may increase risk of hypercoagulability and venous thromboembolism.

Acknowledgment

We thank the staff of Al-Sadr Teaching Hospital and Al-Amal Specialized Hospital for Communicable Diseases in Najaf governorate-Iraq for their help in recruiting patients. We thank the high-skilled staff of the Department of Occupational Safety, Health and the Environment labs of the Imam Ali (PBUH) Holy Shrine, especially Dr. Nawfal Al-Dujaili, for the help in the biomarker assays.

Funding

There was no specific funding for the present study.

Disclosure statement

The authors have no conflict of interest with any commercial or other association connected with the submitted article.

Author's contributions

HA-H and SA-H measured serum biochemical biomarkers. MM performed the statistical analyses. All authors collaborated in the design of the analysis, the discussion of the findings, the drafting and editing of the manuscript, and the final version of the manuscript.

Data Access Statement.

The dataset generated during and/or analyzed during the current study will be available from the corresponding author upon reasonable request and once the dataset has been fully exploited by the authors.

\section{References}

1. Zhou F, Yu T, Du R, Fan G, Liu Y, Liu Z, et al. Clinical course and risk factors for mortality of adult inpatients with COVID-19 in Wuhan, China: a retrospective cohort study. Lancet. 2020;395(10229):1054-62.

2. Tan CW, Tan JY, Wong WH, Cheong MA, Ng IM, Conceicao EP, et al. Clinical and laboratory features of hypercoagulability in COVID-19 and other respiratory viral infections amongst predominantly younger adults with few comorbidities. Scientific Reports. 2021;11(1):1793.

3. Song J-W, Zhang C, Fan X, Meng F-P, Xu Z, Xia P, et al. Immunological and inflammatory profiles in mild and severe cases of COVID-19. Nature communications. 2020;11(1):1-10. 
4. Hasan SS, Capstick T, Ahmed R, Kow CS, Mazhar F, Merchant HA, et al. Mortality in COVID-19 patients with acute respiratory distress syndrome and corticosteroids use: a systematic review and meta-analysis. Expert Rev Respir Med. 2020;14(11):1149-63.

5. Amini Pouya M, Afshani SM, Maghsoudi AS, Hassani S, Mirnia K. Classification of the present pharmaceutical agents based on the possible effective mechanism on the COVID-19 infection. Daru : journal of Faculty of Pharmacy, Tehran University of Medical Sciences. 2020:1-20.

6. Merdji H, Mayeur S, Schenck M, Oulehri W, Clere-Jehl R, Cunat S, et al. Histopathological features in fatal COVID-19 acute respiratory distress syndrome. Med Intensiva. 2021.

7. Java A, Apicelli AJ, Liszewski MK, Coler-Reilly A, Atkinson JP, Kim AH, et al. The complement system in COVID-19: friend and foe? JCI insight. 2020;5(15).

8. Gralinski LE, Sheahan TP, Morrison TE, Menachery VD, Jensen K, Leist SR, et al. Complement activation contributes to severe acute respiratory syndrome coronavirus pathogenesis. MBio. 2018;9(5).

9. Sarma VJ, Huber-Lang M, Ward PA. Complement in lung disease.

Autoimmunity. 2006;39(5):387-94.

10. Dunkelberger JR, Song W-C. Complement and its role in innate and adaptive immune responses. Cell Research. 2010;20(1):34-50.

11. Bosmann M, Ward PA. Role of C3, C5 and anaphylatoxin receptors in acute lung injury and in sepsis. Adv Exp Med Biol. 2012;946:147-59.

12. Ghazavi A, Mosayebi G, Keshavarzian N, Rabiemajd S, Ganji A. Reduction of Inflammatory C3 and C4 Complement Proteins in Severe COVID-19 Patients. 2020. 13. Fang S, Wang H, Lu L, Jia Y, Xia Z. Decreased complement C3 levels are associated with poor prognosis in patients with COVID-19: A retrospective cohort study. International Immunopharmacology. 2020;89:107070.

14. Gavriilaki E, Brodsky RA. Severe COVID-19 infection and thrombotic microangiopathy: success does not come easily. British journal of haematology. 2020;189(6):e227-e30.

15. Satyam A, Tsokos MG, Brook OR, Hecht JL, Moulton VR, Tsokos GC. Activation of classical and alternative complement pathways in the pathogenesis of lung injury in COVID-19. Clinical Immunology. 2021;226:108716.

16. Campbell CM, Kahwash R. Will complement inhibition be the new target in treating COVID-19-related systemic thrombosis? Circulation. 2020;141(22):1739-41.

17. Klok F, Kruip M, Van der Meer N, Arbous M, Gommers D, Kant K, et al. Incidence of thrombotic complications in critically ill ICU patients with COVID-19. Thrombosis research. 2020;191:145-7.

18. Amara U, Flierl MA, Rittirsch D, Klos A, Chen H, Acker B, et al. Molecular Intercommunication between the Complement and Coagulation Systems. The Journal of Immunology. 2010;185(9):5628.

19. Offermanns S. Activation of platelet function through $\mathrm{G}$ protein-coupled receptors. Circ Res. 2006;99(12):1293-304.

20. Ruan K-H. Advance in understanding the biosynthesis of prostacyclin and thromboxane A2 in the endoplasmic reticulum membrane via the cyclooxygenase pathway. Mini reviews in medicinal chemistry. 2004;4(6):639-47. 
21. Ruan $\mathrm{K}-\mathrm{H}$. Thrombogenesis and anti-thrombogenesis in atherosclerosis mediated by prostanoids through the cyclooxygenase pathway: Nova Science Publishers Inc New York; 2005.

22. FitzGerald GA, Oates JA, Hawiger J, Maas RL, Roberts LJ, Lawson J, et al. Endogenous biosynthesis of prostacyclin and thromboxane and platelet function during chronic administration of aspirin in man. The Journal of clinical investigation. 1983;71(3):676-88.

23. Conti P, Caraffa A, Gallenga C, Ross R, Kritas S, Frydas I, et al. IL-1 induces throboxane-A2 (TxA2) in COVID-19 causing inflammation and micro-thrombi: inhibitory effect of the IL-1 receptor antagonist (IL-1Ra). Journal of biological regulators and homeostatic agents. 2020;34(4).

24. Chu L-y, Liou J-Y, Wu KK. Prostacyclin protects vascular integrity via PPAR/14-3-3 pathway. Prostaglandins \& other lipid mediators. 2015;118:19-27.

25. McAdam B, Catella-Lawson F, Mardini I, Kapoor S, Lawson J, FitzGerald G. Systemic biosynthesis of prostacyclin by cyclooxygenase (COX)-2: the human pharmacology of a selective inhibitor of COX-2. Proceedings of the National Academy of Sciences. 1999;96(1):272-7.

26. Cheng Y, Austin SC, Rocca B, Koller BH, Coffman TM, Grosser T, et al. Role of prostacyclin in the cardiovascular response to thromboxane A2. Science. 2002;296(5567):539-41.

27. Fleming I. The factor in EDHF: Cytochrome P450 derived lipid mediators and vascular signaling. Vascular pharmacology. 2016;86:31-40.

28. Vila L. Cyclooxygenase and 5-lipoxygenase pathways in the vessel wall: Role in atherosclerosis. Medicinal research reviews. 2004;24(4):399-424.

29. Manieri NA, Mack MR, Himmelrich MD, Worthley DL, Hanson EM, Eckmann

L, et al. Mucosally transplanted mesenchymal stem cells stimulate intestinal healing by promoting angiogenesis. The Journal of clinical investigation. 2015;125(9):3606-18.

30. Martini R. The compelling arguments for the need for microvascular investigation in COVID-19 critical patients. Clinical hemorheology and microcirculation. 2020(Preprint):1-8.

31. Al-Hakeim HK, Al-Jassas HK, Morris G, Maes M. Increased angiotensinconverting enzyme 2, sRAGE and immune activation, but lowered calcium and magnesium in COVID-19: association with chest CT abnormalities and lowered peripheral oxygen saturation. medRxiv. 2021:2021.03.26.21254383.

32. Huang J, Cheng A, Kumar R, Fang Y, Chen G, Zhu Y, et al. Hypoalbuminemia predicts the outcome of COVID-19 independent of age and co-morbidity. J Med Virol. 2020;92(10):2152-8.

33. Zhang Y, Zheng L, Liu L, Zhao M, Xiao J, Zhao Q. Liver impairment in COVID19 patients: A retrospective analysis of 115 cases from a single centre in Wuhan city, China. Liver Int. 2020;40(9):2095-103.

34. James MT, Zhang J, Lyon AW, Hemmelgarn BR. Derivation and internal validation of an equation for albumin-adjusted calcium. BMC clinical pathology. 2008;8(1):1-6.

35. Chen S, Zhang Z, Wu Y, Shi Q, Yan H, Mei N, et al. Endoplasmic Reticulum Stress and Store-Operated Calcium Entry Contribute to Usnic Acid-Induced Toxicity in Hepatic Cells. Toxicological sciences : an official journal of the Society of Toxicology. 2015;146(1):116-26. 
36. Zhang IX, Ren J, Vadrevu S, Raghavan M, Satin LS. ER stress increases storeoperated $\mathrm{Ca}(2+)$ entry (SOCE) and augments basal insulin secretion in pancreatic beta cells. The Journal of biological chemistry. 2020;295(17):5685-700.

37. Fung TS, Liu DX. Coronavirus infection, ER stress, apoptosis and innate immunity. Frontiers in microbiology. 2014;5(296).

38. Morris G, Athan E, Walder K, Bortolasci CC, O'Neil A, Marx W, et al. Can endolysosomal deacidification and inhibition of autophagy prevent severe COVID-19? Life sciences. 2020;262:118541.

39. Güzel A, Doğan E, Türkçü G, Kuyumcu M, Kaplan İ, Çelik F, et al. Dexmedetomidine and Magnesium Sulfate: A Good Combination Treatment for Acute Lung Injury? J Invest Surg. 2019;32(4):331-42.

40. Abiri B, Vafa M. Effects of vitamin D and/or magnesium supplementation on mood, serum levels of BDNF, inflammatory biomarkers, and SIRT1 in obese women: a study protocol for a double-blind, randomized, placebo-controlled trial. Trials. 2020;21(1):225.

41. Sheu JR, Hsiao G, Shen MY, Lee YM, Yen MH. Antithrombotic effects of magnesium sulfate in in vivo experiments. Int J Hematol. 2003;77(4):414-9.

42. Djagbletey R, Phillips B, Boni F, Owoo C, Owusu-Darkwa E, deGraft-Johnson $\mathrm{PKG}$, et al. Relationship between serum total magnesium and serum potassium in emergency surgical patients in a tertiary hospital in Ghana. Ghana medical journal. 2016;50(2):78-83.

43. Kwee TC, Kwee RM. Chest CT in COVID-19: what the radiologist needs to know. RadioGraphics. 2020;40(7):1848-65.

44. Moustafa HE-DS, El-Seddek M. Accurate diagnosis of covid-19 based on deep neural networks and chest X-ray images.(dept. e (electronics)). Bulletin of the Faculty of Engineering Mansoura University. 2020;45(3):11-5.

45. Marcos-Jiménez A, Sánchez-Alonso S, Alcaraz-Serna A, Esparcia L, López-Sanz C, Sampedro-Núñez M, et al. Deregulated cellular circuits driving immunoglobulins and complement consumption associate with the severity of COVID-19. medRxiv. 2020:2020.06.15.20131706.

46. Delanghe JR, De Buyzere ML, Speeckaert MM. C3 and ACE1 polymorphisms are more important confounders in the spread and outcome of COVID-19 in comparison with ABO polymorphism. European journal of preventive cardiology. 2020;27(12):13312.

47. Qin C, Zhou L, Hu Z, Zhang S, Yang S, Tao Y, et al. Dysregulation of Immune Response in Patients With Coronavirus 2019 (COVID-19) in Wuhan, China. Clin Infect Dis. 2020;71(15):762-8.

48. Peffault de Latour R, Bergeron A, Lengline E, Dupont T, Marchal A, Galicier L, et al. Complement C5 inhibition in patients with COVID-19 - a promising target? Haematologica. 2020;105(12):2847-50.

49. Holter JC, Pischke SE, de Boer E, Lind A, Jenum S, Holten AR, et al. Systemic complement activation is associated with respiratory failure in COVID-19 hospitalized patients. Proc Natl Acad Sci U S A. 2020;117(40):25018-25.

50. Ciceri F, Beretta L, Scandroglio AM, Colombo S, Landoni G, Ruggeri A, et al. Microvascular COVID-19 lung vessels obstructive thromboinflammatory syndrome 
(MicroCLOTS): an atypical acute respiratory distress syndrome working hypothesis. Critical care and resuscitation. 2020;22(2):95.

51. Reilly M, Fitzgerald G. Cellular activation by thromboxane A2 and other eicosanoids. European heart journal. 1993;14:88-93.

52. Gupta A, Kalantar-Zadeh K, Reddy ST. Ramatroban as a Novel Immunotherapy for COVID-19. J Mol Genet Med. 2020;14(3).

53. Wise J. Covid-19 and thrombosis: what do we know about the risks and treatment? : British Medical Journal Publishing Group; 2020.

54. Manne BK, Denorme F, Middleton EA, Portier I, Rowley JW, Stubben C, et al. Platelet gene expression and function in patients with COVID-19. Blood. 2020;136(11):1317-29.

55. Tang N, Li D, Wang X, Sun Z. Abnormal coagulation parameters are associated with poor prognosis in patients with novel coronavirus pneumonia. Journal of thrombosis and haemostasis. 2020;18(4):844-7.

56. Bilaloglu S, Aphinyanaphongs Y, Jones S, Iturrate E, Hochman J, Berger JS. Thrombosis in Hospitalized Patients With COVID-19 in a New York City Health System. Jama. 2020;324(8):799-801.

57. Robb CT, Goepp M, Rossi AG, Yao C. Non-steroidal anti-inflammatory drugs, prostaglandins, and COVID-19. British Journal of pharmacology. 2020;177(21):4899920.

58. Midgett C, Stitham J, Martin KA, Hwa J. Prostacyclin receptor regulation-from transcription to trafficking. Current molecular medicine. 2011;11(7):517-27.

59. Kim S, Kunapuli SP. P2Y12 receptor in platelet activation. Platelets. 2011;22(1):54-8.

60. Toki S, Reiss S, Goleniewska K, Moore ML, FitzGerald G, Peebles RS, Jr. Prostaglandin $\mathrm{I}<\mathrm{sub}>2</$ sub $>$ Receptor (IP) Signaling Inhibits Lung Type I Interferon Expression by RSV Infection. Journal of Allergy and Clinical Immunology. 2013;131(2):AB191.

61. Dorris SL, Peebles RS. PGI2 as a regulator of inflammatory diseases. Mediators of inflammation. 2012;2012.

62. Honda T, Segi-Nishida E, Miyachi Y, Narumiya S. Prostacyclin-IP signaling and prostaglandin E2-EP2/EP4 signaling both mediate joint inflammation in mouse collageninduced arthritis. The Journal of experimental medicine. 2006;203(2):325-35.

63. Nakajima S, Honda T, Sakata D, Egawa G, Tanizaki H, Otsuka A, et al. Prostaglandin I2-IP signaling promotes Th1 differentiation in a mouse model of contact hypersensitivity. The Journal of Immunology. 2010;184(10):5595-603.

64. Canzano P, Brambilla M, Porro B, Cosentino N, Tortorici E, Vicini S, et al. Platelet and Endothelial Activation as Potential Mechanisms Behind the Thrombotic Complications of COVID-19 Patients. JACC Basic Transl Sci. 2021;6(3):202-18.

65. Perico L, Benigni A, Casiraghi F, Ng LFP, Renia L, Remuzzi G. Immunity, endothelial injury and complement-induced coagulopathy in COVID-19. Nature Reviews Nephrology. 2021;17(1):46-64.

66. Magro C, Mulvey JJ, Berlin D, Nuovo G, Salvatore S, Harp J, et al. Complement associated microvascular injury and thrombosis in the pathogenesis of severe COVID-19 infection: a report of five cases. Translational Research. 2020;220:1-13. 
67. Ackermann M, Verleden SE, Kuehnel M, Haverich A, Welte T, Laenger F, et al. Pulmonary vascular endothelialitis, thrombosis, and angiogenesis in Covid-19. New England Journal of Medicine. 2020;383(2):120-8.

68. Teuwen L-A, Geldhof V, Pasut A, Carmeliet P. COVID-19: the vasculature unleashed. Nature Reviews Immunology. 2020;20(7):389-91.

69. Varga Z, Flammer AJ, Steiger P, Haberecker M, Andermatt R, Zinkernagel AS, et al. Endothelial cell infection and endotheliitis in COVID-19. The Lancet. 2020;395(10234):1417-8.

70. Golshiri K, Ataei Ataabadi E, Portilla Fernandez EC, Jan Danser AH, Roks AJM. The importance of the nitric oxide-cGMP pathway in age-related cardiovascular disease: Focus on phosphodiesterase- 1 and soluble guanylate cyclase. Basic Clin Pharmacol Toxicol. 2020;127(2):67-80.

71. Maes M. A review on the acute phase response in major depression. Reviews in the neurosciences. 1993;4(4):407-16.

72. Soeters PB, Wolfe RR, Shenkin A. Hypoalbuminemia: pathogenesis and clinical significance. Journal of Parenteral and Enteral Nutrition. 2019;43(2):181-93.

73. Ronit A, Kirkegaard-Klitbo DM, Dohlmann TL, Lundgren J, Sabin CA, Phillips AN, et al. Plasma albumin and incident cardiovascular disease: results from the CGPS and an updated meta-analysis. Arteriosclerosis, thrombosis, and vascular biology. 2020;40(2):473-82.

74. Thachil J, Tang N, Gando S, Falanga A, Cattaneo M, Levi M, et al. ISTH interim guidance on recognition and management of coagulopathy in COVID-19. Journal of Thrombosis and Haemostasis. 2020;18(5):1023-6.

75. Paar M, Rossmann C, Nusshold C, Wagner T, Schlagenhauf A, Leschnik B, et al. Anticoagulant action of low, physiologic, and high albumin levels in whole blood. PLoS One. 2017;12(8):e0182997.

76. Manne BK, Denorme F, Middleton EA, Portier I, Rowley JW, Stubben C, et al. Platelet gene expression and function in patients with COVID-19. Blood, The Journal of the American Society of Hematology. 2020;136(11):1317-29.

77. Yadav H, Kor DJ. Platelets in the pathogenesis of acute respiratory distress syndrome. American Journal of Physiology-Lung Cellular and Molecular Physiology. 2015;309(9):L915-L23.

78. Middleton EA, Weyrich AS, Zimmerman GA. Platelets in pulmonary immune responses and inflammatory lung diseases. Physiological reviews. 2016;96(4):1211-59.

79. Koupenova M, Clancy L, Corkrey HA, Freedman JE. Circulating Platelets as Mediators of Immunity, Inflammation, and Thrombosis. Circ Res. 2018;122(2):337-51. 80. Ma K-L, Liu Z-H, Cao C-F, Liu M-K, Liao J, Zou J-B, et al. COVID-19 Myocarditis and Severity Factors: An Adult Cohort Study. medRxiv. 2020:2020.03.19.20034124.

81. Yang C, Ma X, Wu J, Han J, Zheng Z, Duan H, et al. Low serum calcium and phosphorus and their clinical performance in detecting COVID-19 patients. J Med Virol. 2021;93(3):1639-51.

82. Torres B, Alcubilla P, González-Cordón A, Inciarte A, Chumbita M, Cardozo C, et al. Impact of low serum calcium at hospital admission on SARS-CoV-2 infection outcome. International Journal of Infectious Diseases. 2021;104:164-8. 
83. Di Filippo L, Formenti AM, Rovere-Querini P, Carlucci M, Conte C, Ciceri F, et al. Hypocalcemia is highly prevalent and predicts hospitalization in patients with COVID-19. Endocrine. 2020;68(3):475-8.

84. Guo L, Ren L, Yang S, Xiao M, Chang D, Yang F, et al. Profiling Early Humoral Response to Diagnose Novel Coronavirus Disease (COVID-19). Clin Infect Dis. 2020;71(15):778-85.

85. Zhou P, Yang X-L, Wang X-G, Hu B, Zhang L, Zhang W, et al. A pneumonia outbreak associated with a new coronavirus of probable bat origin. nature. 2020;579(7798):270-3.

86. Maeda K, Mehta H, Drevets DA, Coggeshall KM. IL-6 increases B-cell IgG production in a feed-forward proinflammatory mechanism to skew hematopoiesis and elevate myeloid production. Blood, The Journal of the American Society of Hematology. 2010;115(23):4699-706.

87. Nielsen C, Fischer E, Leslie R. The role of complement in the acquired immune response. Immunology. 2000;100(1):4.

88. Zeng F, Dai C, Cai P, Wang J, Xu L. Jianyu Li1, Guoyun Hu1, LW (2020). A comparison study of SARS-CoV-2 IgG antibody between male and female COVID-19 patients: a possible reason underlying different outcome between gender. BMJ, February 2019.1-13. 